\title{
Experimental analysis of the optical spectra of directionally bistable semiconductor ring lasers
}

\author{
Maria Jose Latorre Vidal ${ }^{\mathrm{a}}$, Sandor Furst ${ }^{\mathrm{b}}$, Gabor Mezosi ${ }^{\mathrm{b}}$, Marco Zanola ${ }^{\mathrm{a}}$, Marc Sorel ${ }^{\mathrm{b}}$, \\ Antonio Perez ${ }^{\mathrm{c}}$, Alessandro Scirè ${ }^{\mathrm{c}}$, Salvador Balle ${ }^{\mathrm{c}}$, Guido Giuliani ${ }^{\mathrm{a}}$ \\ ${ }^{a}$ Dipartimento di Elettronica, Università di Pavia, via Ferrata 1, I-27100 Pavia, Italy \\ ${ }^{\mathrm{b}}$ Department of Electronics and Electrical Engineering, University of Glasgow, \\ G12 8LT, Glasgow, United Kingdom \\ ${ }^{\mathrm{c}}$ Instituto de Fisica Interdisciplinar y Sistemas Complejos, IFISC, CSIC-UIB, Campus UIB, \\ Ctra. Valldemossa km. 7.5, E-07122 Palma de Mallorca, Spain.
}

\begin{abstract}
The optical spectrum of monolithic Semiconductor Ring Lasers (SRLs) is measured simultaneously for both lasing directions with a grating-based OSA, in the regimes of bidirectional and unidirectional operation. In the unidirectional operation regime the SMSR is larger than $25 \mathrm{~dB}$, and the directional extinction ratio (i.e., the ratio of the power emitted in the two opposite directions) is larger than $20 \mathrm{~dB}$. The influence of the current injected in the active output waveguides that act as SOAs is outlined. In the unidirectional regime the linewidth of the SRL is measured by an heterodyne technique, revealing linewidth values around $2 \mathrm{MHz}$.
\end{abstract}

Keywords: Semiconductor ring laser, laser linewidth, laser dynamics, laser model.

\section{INTRODUCTION}

Semiconductor ring lasers (SRLs) are attractive devices because they can be fabricated without the need for cleaved facet mirrors ${ }^{1-3}$, offering the possibility to be monolithically integrated with other optoelectronic devices. SRLs can support two counter propagating lasing modes, that give rise to a rich phenomenology of operating regimes and dynamics. In particular, SRLs have been demonstrated to operate uni-directionally ${ }^{4}$ with the possibility to select the active mode by means of electronic control, and also bi-directionally, either in continuous wave $(\mathrm{CW})$ or in an interesting alternate oscillation regime 5 .

Potential application for SRLs range from inertial rotation sensors to photonic devices capable of performing high-speed all-optical signal processing operations. Recently, a device combining two coupled micro-ring laser cavities with an area of $20 \times 40$ micron squared was demonstrated as ultrafast all-optical switch and optical memory ${ }^{6}$. And single-ring devices with small radius have been demonstrated to show directional bistability ${ }^{7}$.

It is of interest to experimentally investigate the spectral properties of SRLs, with the aim of assessing their performance as light sources for optical communication systems.

In this work, the optical spectrum of monolithic SRLs is measured simultaneously for both lasing directions using a grating-based OSA, in the regimes of bidirectional and unidirectional operation. The influence of the current injected in the active output waveguides is outlined. In the unidirectional regime, the linewidth of the SRL is measured by an heterodyne technique, revealing linewidth values around $2 \mathrm{MHz}$.

\section{SEMICONDUCTOR RING LASER DEVICE}

Monolithic racetrack shaped SRL devices were fabricated at Glasgow University in a InGaAs/InGaAlAs/InP MQW material. The device structure is shown in Fig. 1. The SRL has two semi-circular sections with $150 \mu \mathrm{m}$ radius connecting two straight sections of $200 \mu \mathrm{m}$ length that are coupled to two straight output waveguides. Thus, the device has four input/output ports. In each of the two output waveguides, two semiconductor optical amplifiers (SOAs) are defined by

$\S$ e-mail address: guido.giuliani@unipv.it

Semiconductor Lasers and Laser Dynamics III, edited by Krassimir P. Panajotov, Marc Sciamanna, Angel A. Valle, Rainer Michalzik, Proc. of SPIE Vol. 6997, 699725, (2008) · 0277-786X/08/\$18 · doi: 10.1117/12.783929 


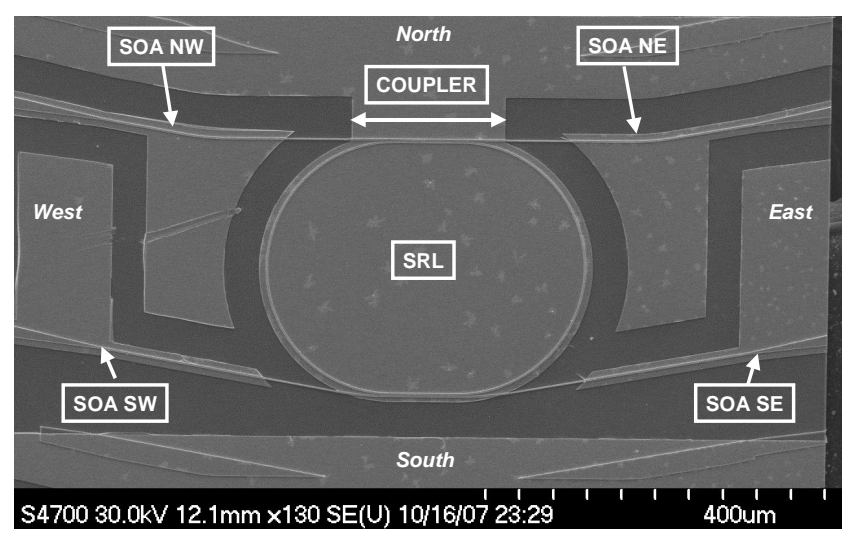

Figure 1. Layout of SRL device.

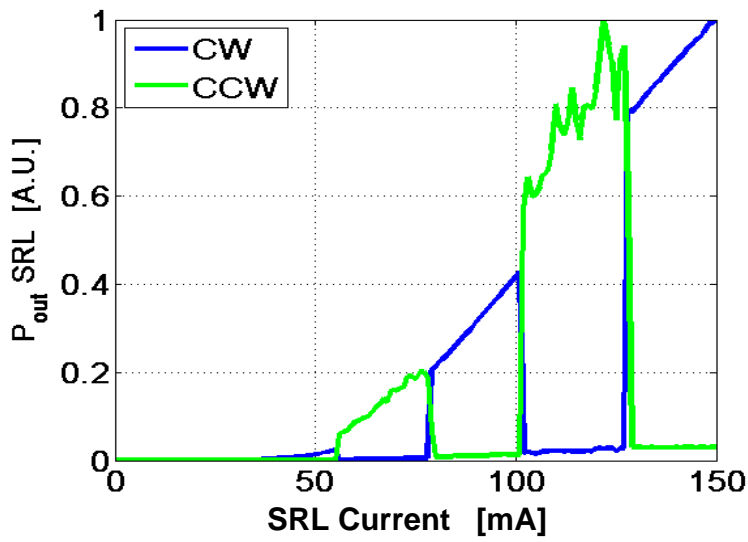

Figure 2. P-I curves for the two counterpropagating modes measured using lensed fibers.

separate electrical contacts. To identify the four outputs and SOAs we use the North-South-East-West notation (see Fig. $1)$.

The SRLs operate CW at room temperature, with threshold currents between 35 and $60 \mathrm{~mA}$, emitting up to $3 \mathrm{~mW}$ at $\lambda=1550-1570 \mathrm{~nm}$. Fig. 2 shows a typical P-I curve measured for the two counterpropagating modes $(\mathrm{CW}=$ clockwise; $\mathrm{CCW}=$ counter-clockwise) using a lensed optical fibre. A bidirectional regime (i.e., $\mathrm{CW}$ and $\mathrm{CCW}$ modes both active) appears just above threshold, and as the current is increased a neat unidirectional regime takes place, where only one directional mode is active and the other is highly suppressed. This is a consequence of the optical directional bistability intrinsic to all-active SRLs, that occurs thanks to the non-linear cross-gain saturation in the active medium ${ }^{5}$. This bistability is observed in all fabricated devices and it is extremely robust. Directional switchings are observed as the current is increased, probably caused by relative changes of the phase conditon between the two counterpropagating modes. These phase changes are caused by thermal effects.

When the SRL is operated in the unidirectional bistable regime, the device can be used as an optical digital memory cell, capable of storing one bit of information. The status of the memoory depends on the particular lasing direction. The direction of operation (and thus the status of the memory cell) can be changed by injecting an external optical signal into

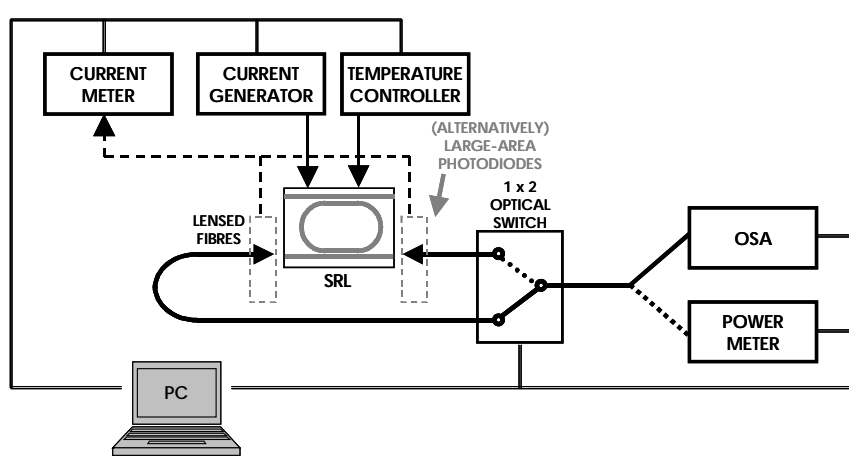

Figure 3. Experimental set-up for spectral characterization.

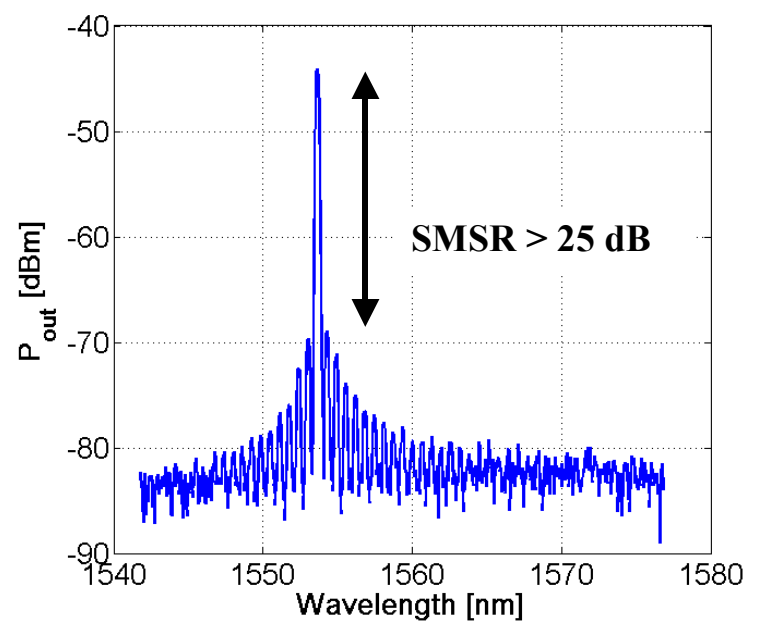

Figure 4. Example of optical spectrum taken at high current. 
one of the input/output ports, so that the injected signal propagates within the ring cavity in the opposite direction with respect to the lasing mode ${ }^{8}$. If the injected power exceeds a certain threshold level, photons travelling in the new direction induce a regenerative directional switching that reverts the state of the memory. The new lasing direction is maintained after the external triggering light is switched off. Hence, the SRL operates as an all-optical Set-Reset FlipFlop (SR-FF).

\section{BIDIRECTIONAL SPECTRAL MEASUREMENTS BY GRATING-BASED OSA}

The spectral measurement are carried out by a grating-based OSA with $0.01 \mathrm{~nm}$ resolution using the set-up shown in Fig. 3. Lensed fibers are used to couple light out of the device (from North waveguides), and an opto-mechanical fiber optic switch is used to select one of the SRL outputs. During the measurement, that is assisted by a PC, the SRL current is increased, and the optical spectrum is maesured from each direction.

Figure 4 reports a single spectrum taken at high current (in unidirectional regime), where it is clear that the SRL exhibits a high side-mode suppression ratio (SMSR).

Figure 5 reports the complete characterization performed for a value of the currents injected into North-West and NorthEast SOAs equal to $2.5 \mathrm{~mA}$. The SRL emits a up tp a few $\mathrm{mW}$ in air, and the power coupling efficiency into the fiber can

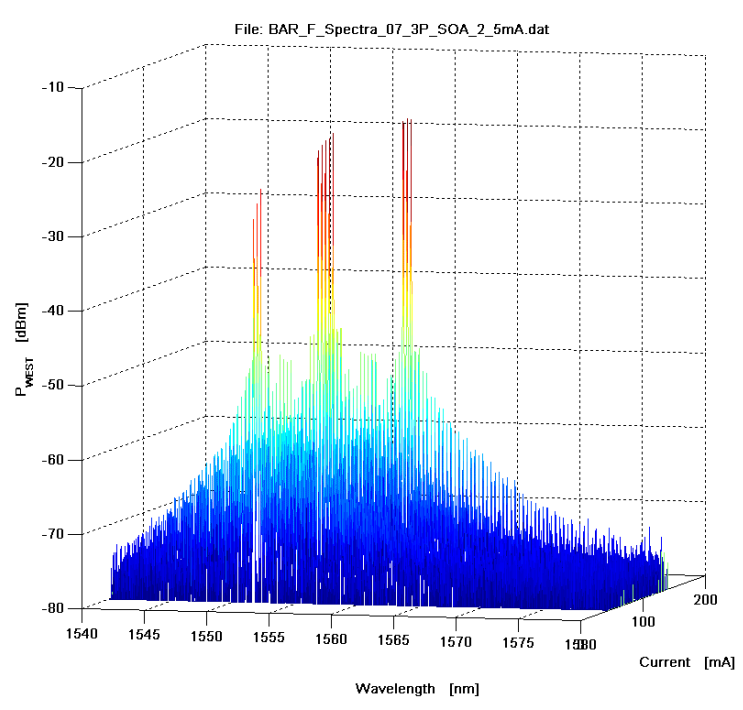

(a)

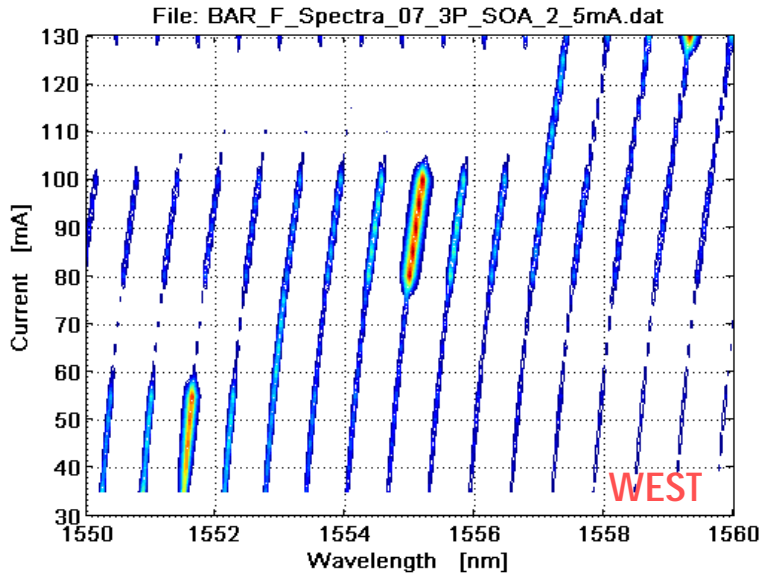

(c)

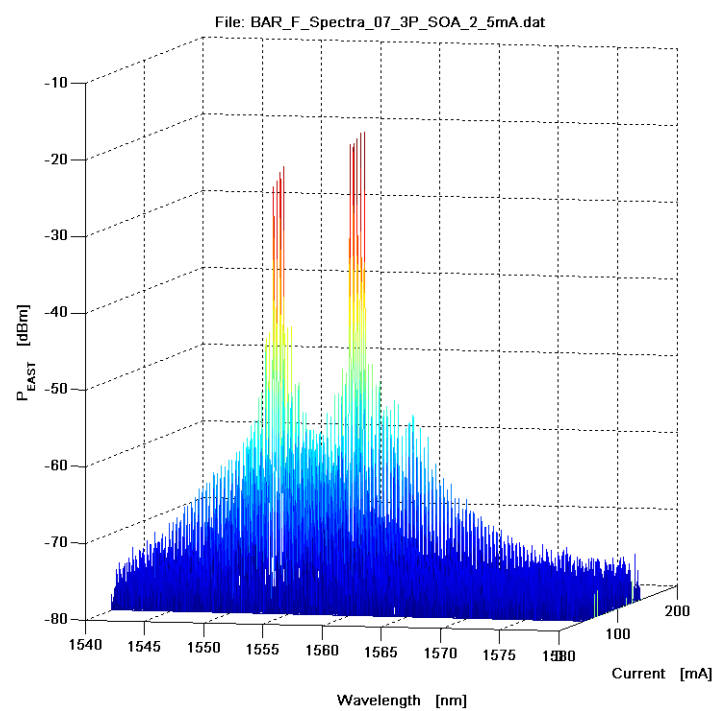

(b)

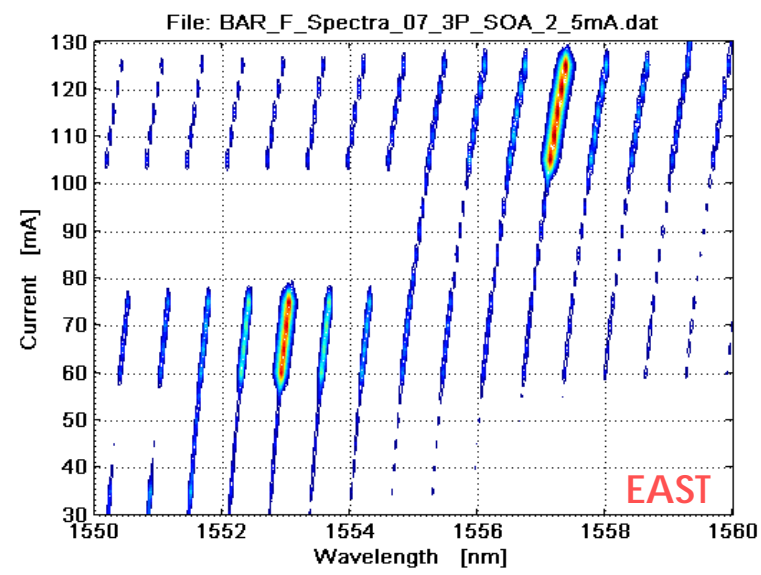

(d)

Figure 5. Spectra measured as a function of the curent injected into the ring contact, for the West direction (a and c), and for the East direction ( $b$ and $d)$. In the contour plots ( $c$ and $d$ ) level lines are separated by $3 \mathrm{~dB}$. The current injected into the Noth-West and the North-East SOAs is $2.5 \mathrm{~mA}$ 

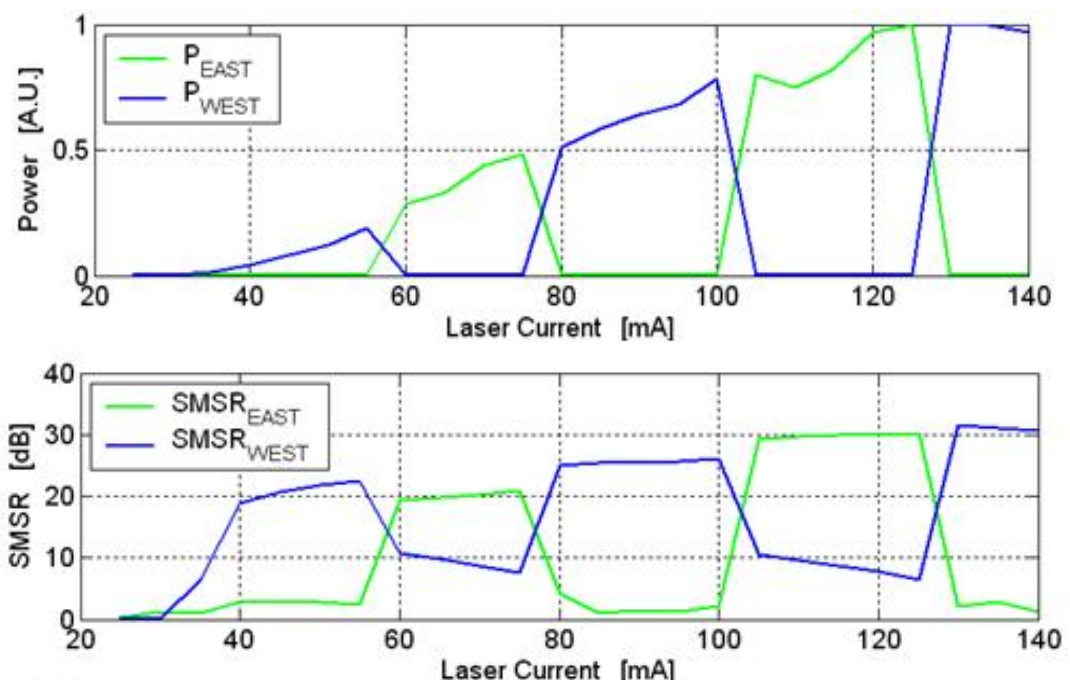

(a)

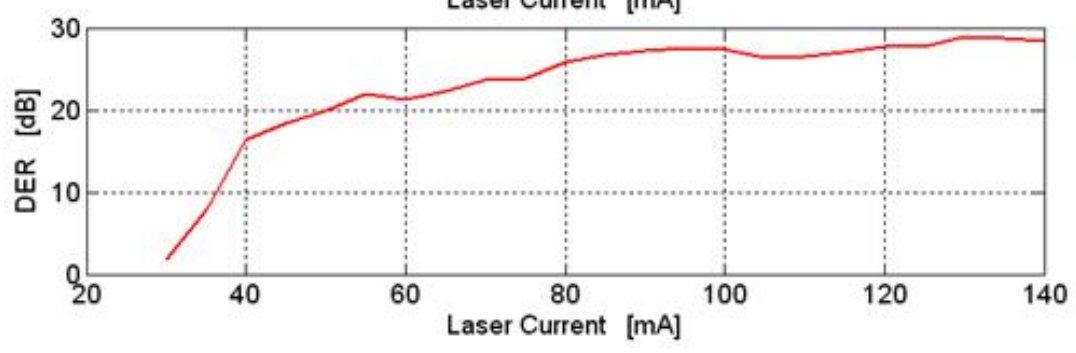

(c)

Figure 6. (a) P-I curve as calculated from the measured spectra. The current step is $5 \mathrm{~mA}$, which accounts for the apparent smoothness of directional switchings. (b) SMSR as a function of the injected ring current. (c) DER (directional extinction ratio) as a function of the injected ring current. The current injected into the North-West and the North-East SOAs is $2.5 \mathrm{~mA}$ for all graphs.

be between $10 \%$ and $40 \%$ when the coupling is optimized (and we notice that the efficiency was not maximized in the present measurements).

Fig. 5a and 5b show a 3D plot of the spectra, respectively for the West and East outputs. Fig. 5c and 5d show a contour plot of the spectra, where the distance between each line corresponds to $3 \mathrm{~dB}$ difference. This SRL has a threshold current of $30 \mathrm{~mA}$. From just above threshold, the SRL operates in the unidirectional regime, with a good suppression of side-modes. Form Fig. 5c and 5d it can be noticed that the longitudinal mode structure undergoes a red-shift because of thermal heating.

Figure 6 reports the following plots: the total power emitted from the two North outputs (Fig. 6a, in normalized arbitrary units); the SMSR (Fig. 6b); the directional extinction ratio, DER (Fig 6c). The DER is defined as the ratio beween the power emitted from one direction and the power emitted in the opposite direction. As the fiber coupling efficiencies during the experiment can be different for the two sides, the DER is calculated after a normalizaton of the powers emitted in the two directions just above threshold.

The results of Fig. 5 and 6 confirm that the SRL spectral properties are indeed good, as the laser emits in a single longitudinal mode for all the values of the injected current. In particular, the SMSR exceeds $30 \mathrm{~dB}$ at high powers, and it is larger than $20 \mathrm{~dB}$ as soon as the current exceeds the threshold value by more than $10 \mathrm{~mA}$. For each lasing direction, the difference in the SMSR when the specific directional mode is active or suppressed is between 10 and $25 \mathrm{~dB}$.

The DER is a crucial parameter for a proficient use of the SRL as all-optical set-reset flip-flop ${ }^{8}$. In the measured device the DER is larger than $20 \mathrm{~dB}$ for ring currents larger than $50 \mathrm{~mA}$.

Figures 7 and 8 report the spectral characterization for larger values of the currents injected into the terminal SOAs, namely 5 and $10 \mathrm{~mA}$. In both cases the unidirectionality is preserved, but for the $10 \mathrm{~mA}$ case no spontaneous directional switching occurs, as the active lasing direction is always West. This has no influence on the use of the SRL as all-optical flip-flop, because the active direction can still be reversed by injecting a proper external optical signal ${ }^{8}$. A possible reason for the absence of spontaneous directional switchings is the increased effective reflection form the device end 


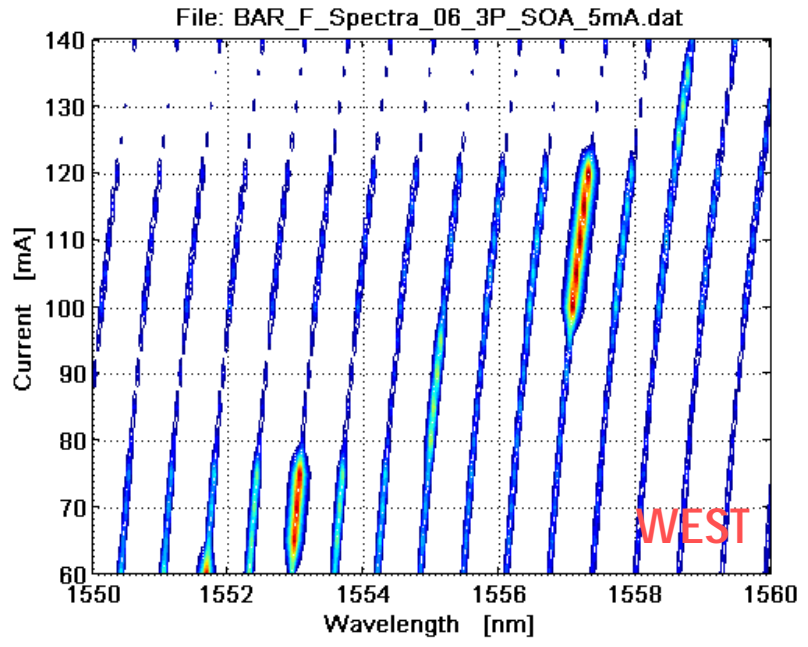

(a)

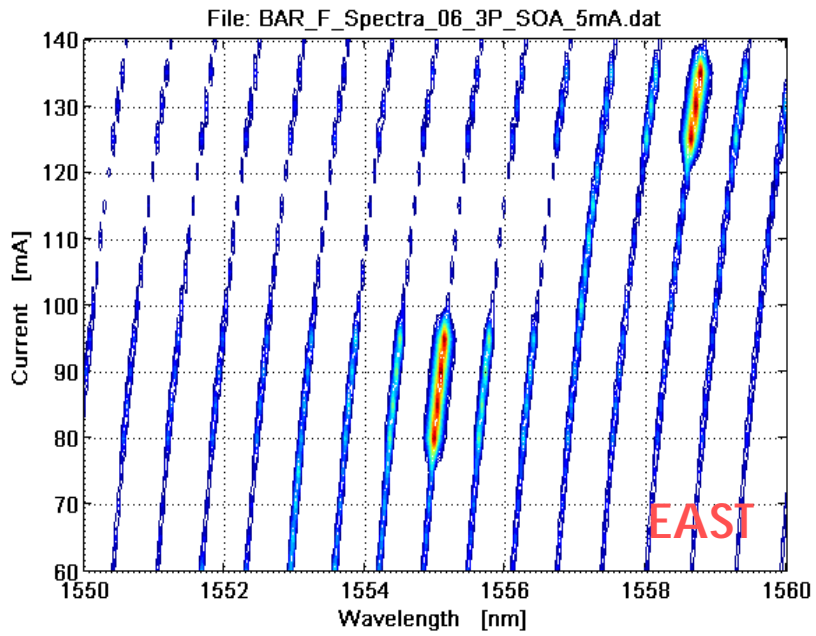

(b)

Figure 7. Spectral contour plots for an injected current into the North-West and the North-East SOAs of 5.0 mA

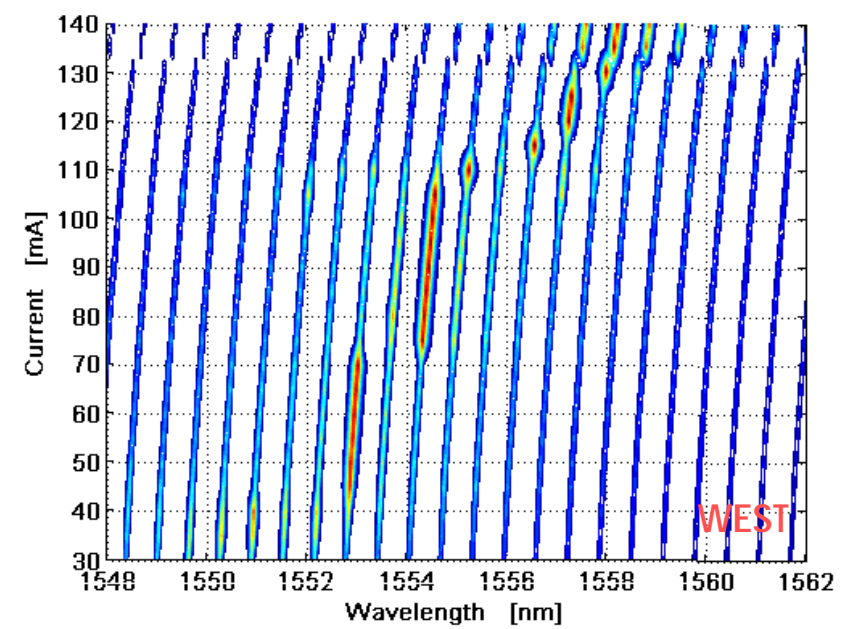

(a)

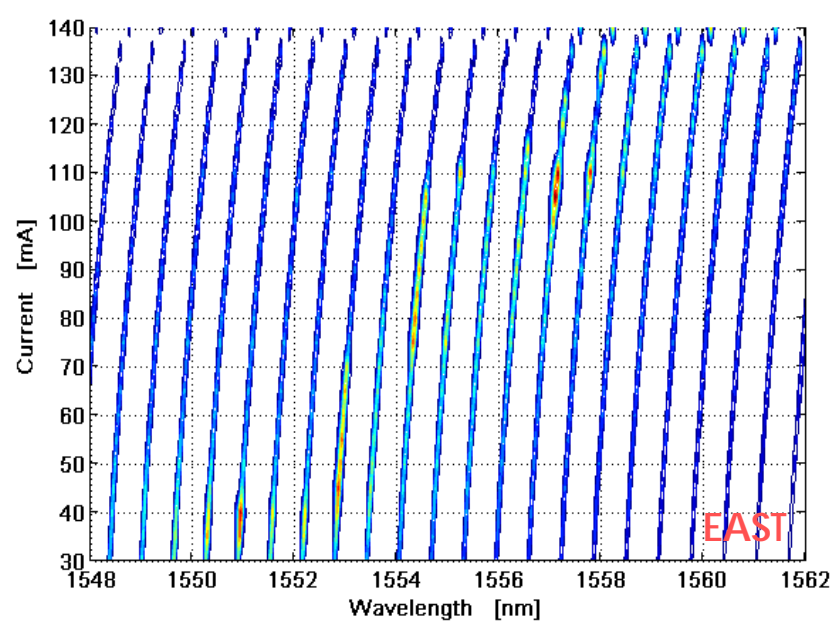

(b)

Figure 8. Spectral contour plots for an injected current into the North-West and the North-East SOAs of $10.0 \mathrm{~mA}$

facets, due to the increased optical gain in the SOAs. The reflections from the facets generate a coupling of one directional mode into the opposite one. As the real device is in practice asymmetric, one of the directional modes experiences a stronger injection from the other, and it is thus enhanced, being the only active lasing direction mode once the SRL enters the unidirectional regime.

\section{LINEWIDTH MEASUREMENTS BY HETERODYNE TECHNIQUE}

The linewidth of the SRL is measured using the hetorodyne method and the experimental set-up shown in Fig. 9a. Due to thermal instabilities, the $-3 \mathrm{~dB}$ linewidth was extrapolated from measurements of the beating spectral width taken at $20 \mathrm{~dB}$ or $-30 \mathrm{~dB}^{9}$. The measured linewidth as a function of the "above-threshold" current is displayed in Fig. 9b. Well above threshold, the linewidth has an average value of about $2.4 \mathrm{MHz}$.

This linewidth value is indeed good, and it confirms that SRLs are good light sources under all aspects related to spectral and coherence properties. No linewidth rebroadening is observed at high currents.

\section{CONCLUSIONS}

We have have shown experimenatlly that a monolithic semiconductor ring laser can have excellent spectral properties, because it emits on a single-longitudinal mode in almost all operating conditions, with a SMSR that can be as large as 


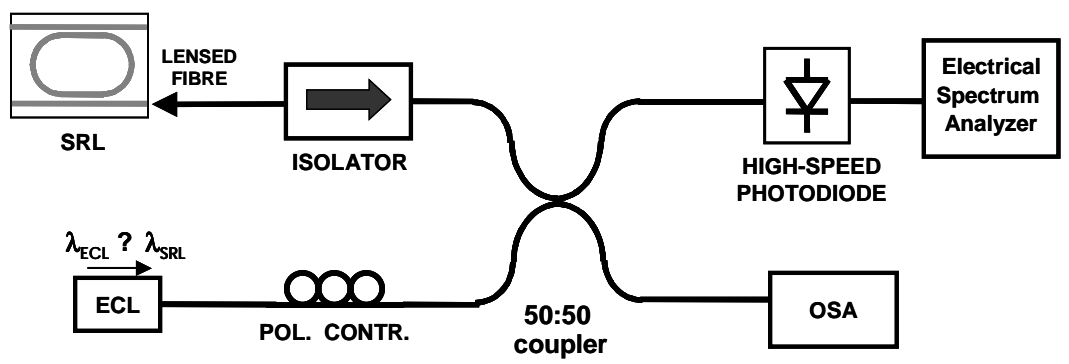

(a)

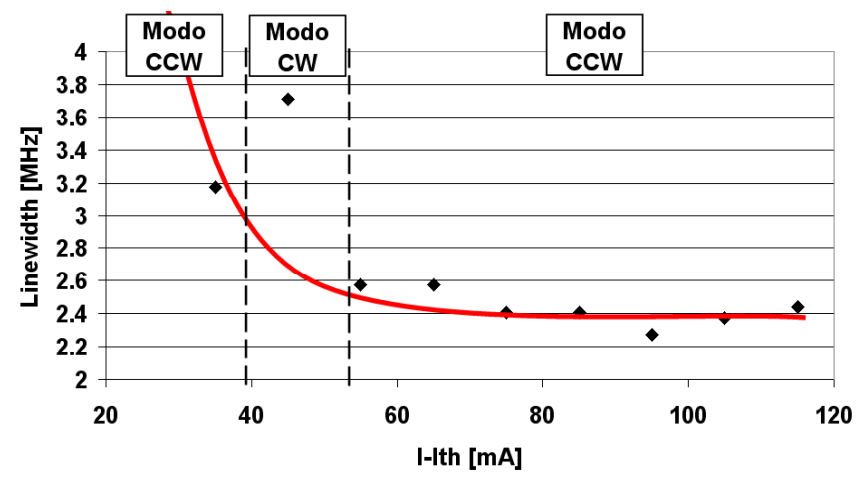

(b)

Figure 9. (a) Experimental set-up for heterodyne linewidth measurement. (b) Measured linewidth as a function of SRL current..

$30 \mathrm{~dB}$. We have also measured the directional extinction ratio (DER), which is of major importance for the applicaton as all-optical flip-flop. The reported DER is larger than $20 \mathrm{~dB}$.

Finally the linewidth of the SRL is around $2.4 \mathrm{MHz}$.

\section{ACKNOWLEDGEMENTS}

This research was founded by he EU-FP6 'IOLOS' project.

\section{REFERENCES}

1. T. F. Krauss, P. J. R. Laybourn and J. S. Roberts, "CW operation of semiconductor ring lasers", Electron. Lett., Vol.26, pp.2095-2097, 1990

2. T. F. Krauss, R. M. De La Rue, P. J. R. Laybourn, B. Vogele, and C. R. Stanley, "Efficient semiconductor ring lasers made by a simple self-aligned fabrication process", IEEE J. Select. Topics Quantum Electron., Vol.1, pp. 757-761, 1995 3. J. P. Hoimer, and G. A. Vawter, "Unidirectional semiconductor ring laser with racetrack cavities", Appl. Phys. Lett., Vol.63, pp. 2457-2459, 1993

4. M. Sorel, G. Giuliani, S. Donati, P. J. R. Laybourn, "Unidirectional bistability in semiconductor waveguide ring lasers", Appl. Phys. Lett., vol.80, pp. 3051-3053, 2002

5. M. Sorel, G. Giuliani, A. Scirè, R. Miglierina, S. Donati, P. J. R. Laybourn, "Operating Regimes of GaAs-AlGaAs Semiconductor Ring Lasers: Experiment and Model", IEEE Journal of Quantum Electronics, vol. 39, n. 10, pp. 11871195,2003

6. M. T. Hill, H. J. S. Dorren, T. De Vries, X. J. M. Leijtens, J. H. Den Besten, B. Smalbrugge, Y.-S. Oei, H. Binsma, G.D. Khoe, M. K. Smit, "A fast low-power optical memory based on coupled micro-ring lasers", Nature, vol. 432, pp. 206209, 2004

7. Z. Wang, G. Verschaffelt, Y. Shu, G. Mezosi, M. Sorel, J. Danckaert, and S. Yu, "Integrated small-sized semiconductor ring laser with novel retro-reflector cavity", IEEE Photon. Technol .Lett., vol. 20, no. 2, pp. 99-101, 2008. 8. G. Giuliani, S. Yu, Z, Wang, G. Yuan, B. Li, I.M. Memon, S. Furst, and M. Sorel, "All-Optical Flip-Flop and Digital Inverter Functions using a Monolithic Semiconductor Ring Laser", ECOC 2007

9. D. Derickson, "Fiber optic test and measurement”, Prentice Hall, Upper Saddle River, N.J., 1998 